\title{
Del éxito en televisión a la participación en las redes sociales. El Príncipe y Galerías Velvet en Facebook ${ }^{1}$
}

\section{From success on TV to involvement in social networks. El Príncipe \& Galerias Velvet on Facebook}

\author{
Leire Gómez Rubio. Universidad de Valladolid
}

Nereida López Vidales. Universidad de Valladolid

Recibido: 30-XI-2014 - Aceptado: 19-II-2015

Resumen:

Este artículo presenta los resultados del análisis de la estrategia televisiva y la actividad participativa del público que registran los perfiles oficiales de Facebook de las dos series de ficción nacional con mayor índice de audiencia durante el primer semestre de 2014: El Príncipe y Galerías Velvet. La investigación pone de manifiesto que los seguidores sociales de ambas ficciones prefieren participar en la red durante el espacio de tiempo más próximo a su proyección en televisión, reaccionando, sobre todo, ante aquellos contenidos de carácter audiovisual.

Palabras clave:

Audiencia, Facebook, televisión social, redes sociales, series de ficción.

Abstract:

The next article shows the results of the analysis on television strategy and participative activity of the public registered by the official profiles on Facebook of the two national series that showed the highest audience average during the first semester of 2014: El Principe and Galerias Velvet. Researches show the preference of the audience on participating on the net during the most approximated time lapse to its broadcasting on television, reacting, above all, to media contents.

Keywords:

Audience, Facebook, social television, social network, fiction-series.

El trabajo se enmarca dentro de la investigación “La contribución juvenil a la conformación de la radio y la televisión social”, realizada por el Observatorio del Ocio y el Entretenimiento Digital-OCENDI (Cod. OCENDI4/1315), financiada por el Observatorio del Ocio y el Entretenimiento Digital y Underwood Comunicación, S.L.U. 


\section{Introducción}

La participación de la audiencia en contenidos de televisión a través de las redes sociales se ha convertido en los últimos años en una práctica habitual. Muestra de ello es que si bien antes la presencia de los distintos canales de televisión en estas plataformas virtuales sociales consistía, de forma mayoritaria, en un único perfil por canal en diferentes redes sociales, poco a poco han aumentado los perfiles destinados a programas determinados. Se trata, en este caso, de actuaciones "que responden al seguimiento de un programa o serie de ficción específica por parte de sus telespectadores, que desean estar informados en todo momento de lo que sucede en relación con el rodaje de dicho contenido, sus rostros de canal, su emisión o cualquier tipo de noticia relacionada con el mismo" (García Mirón, 2011: 90-91).

Se pone así de manifiesto que las necesidades e intereses de la audiencia han cambiado. La figura del prosumidor se consolida, y los programas de televisión ya no cuentan con una única audiencia, la convencional, sino que ahora también disponen de una audiencia social, la derivada de las extensiones de la web 2.0, entre cuyas principales características se encuentra que la práctica cultural de "ver la televisión", hasta hace unos años ritual y predeterminada a un 'aquí y ahora' concretos, se haya transmutado (Barrientos Bueno, 2013), además de poner al alcance de la audiencia la posibilidad de participar activamente en prácticamente todos los programas presentes en las parrillas de televisión.

Una de las consecuencias de esta tendencia es que,

"la industria televisiva se interesa cada vez más por la audiencia social. Para ello, las principales cadenas del país han solicitado a sus departamentos multimedia que adapten sus contenidos a las redes sociales con el objetivo de promocionar sus formatos, así como atraer al mayor número de usuarios a través del 'boca a boca' o el 'muro a muro' que suponen estas plataformas sociales, un punto de encuentro en el que los usuarios pueden compartir opiniones e interactuar con los profesionales de la televisión" (Alonso González, 2014).

El resultado de ello ha sido la proliferación de páginas oficiales en distintas redes sociales de buena parte de los programas que llenan las parrillas televisivas con el claro propósito, por parte de sus promotores, de crear comunidades virtuales con las que a través de la conversación bidireccional que éstas posibilitan se incremente la audiencia de los contenidos, se alimente un sentimiento de compromiso con ese contenido, permita conocer a los usuarios y ofrezca la posibilidad de experimentar otros modelos de negocio y de ingresos económicos, más allá del proporcionado por la publicidad de la televisión convencional (Arrojo, 2013).

Fruto de estas posibilidades, cada vez son más los espacios de televisión que llegan a las redes sociales antes incluso de su estreno en televisión, alentando y construyendo con ello una audiencia social que tiene la posibilidad de conocer, participar e intercambiar contenidos con otros miembros de esa comunidad de seguidores que se conforma en torno a diferentes contenidos audiovisuales. 
Sin embargo, ¿̇cuál es la respuesta de la audiencia social a las estrategias desarrolladas por los canales de televisión en las redes sociales? $i$ Existe realmente una audiencia social que comente, comparta e intercambie contenidos durante la emisión de los programas de televisión? ¿ O se trata de una audiencia que participa en diferido? Para dar respuesta a éstas y otras preguntas, en las siguientes páginas se exponen los resultados de un estudio sobre la actividad y la participación en Facebook que, tanto canales de televisión como audiencia, han realizado en dos series de ficción que durante el primer semestre de 2014 fueron líderes de audiencia en sus días de emisión en televisión. Se trata de El Príncipe y Galerías Velvet, dos ficciones programadas en las noches de los lunes y los martes durante los meses de febrero, marzo, abril y mayo. La elección de ambas ficciones se debe a los altos índices de audiencia registrados por ambas, con una cuota de pantalla entre el 20 y el 33\% en todos sus capítulos (Barlovento Comunicación, 2014).

Aunque Twitter está considerada la red social con mayor vinculación práctica con el sector de la televisión, debido a que su uso está normalizado en el momento de la emisión en directo (Gallego, 2013:15), en este trabajo se ha optado por el análisis de Facebook debido a que, actualmente, es la red social con mayor número de seguidores en España: doce millones de usuarios diarios en junio de 2014, según datos proporcionados por Leverage New Age Media. Asimismo, a pesar de la proliferación de redes sociales experimentada en los últimos años, "en el contexto español Facebook se ve tímidamente amenazada, aunque muy de lejos aún, por Twitter y por la aplicación de móvil WhatsApp” (Parra, Gordo y D’Antonio, 2014: 197). Además, en el caso de las series en este trabajo analizadas, las dos iniciaron su actividad en Facebook con perfiles propios varios meses antes de su llega a la pequeña pantalla.

El objetivo principal de esta aportación es determinar cómo actúan los canales de televisión responsables de la emisión de ambas series a la hora de publicar contenidos en las páginas oficiales de Facebook de sendos espacios, así como observar la actividad que dichas actuaciones provocan en los seguidores sociales de las dos ficciones nacionales.

La hipótesis de partida es que los contenidos compuestos por texto e imagen -tanto vídeo como fotografía- son los preferidos por parte de ambos espacios en Facebook, siendo estos los que acumulan el mayor número de 'like' y de comentarios, así como los más compartidos por sus seguidores sociales. También se parte de la idea de que este tipo de contenidos aumentan su presencia en la red social en las jornadas en las que se emiten las series en televisión, mientras que el resto de días, los canales optan por otro tipo de contenidos, como los compuestos únicamente por texto o imagen.

\subsection{Redes sociales y televisión}

La participación en redes sociales y/o profesionales fue uno de los principales usos que los internautas hicieron de la triple $W$ en España durante 2013. Concretamente, un 48\% de los usuarios de Internet participaron en este tipo de plataformas virtuales, el mismo porcentaje que aquellos que emplearon la Red para buscar información sobre bienes y servicios,y cifra 
únicamente superada, con un $62 \%$, por quienes señalaron emplear Internet, sobre todo, para enviar y recibir $e$-mails (ONTSI, 2014: 50-51).

No obstante, la finalidad y la motivación de un internauta cuando opta por hacer uso de una o varias redes sociales no siempre es la misma, igual que los medios también optan por la puesta en marcha de diferentes estrategias. De hecho, la presencia de los diferentes programas de televisión en las redes sociales ha permitido, por un lado, que los medios hayan "multiplicado exponencialmente sus canales de distribución sin coste alguno", además de poder ser utilizadas "para potenciar la marca y fidelizar audiencias" (Ferreras Rodríguez, 2011). Asimismo, junto a las estrategias de promoción puestas en marcha por las diferentes empresas audiovisuales, las redes sociales también han derivado, dentro de este ecosistema mediático, hacia una "vertiente comunitaria", donde "el usuario puede comunicarse e interactuar con la empresa que le proporciona el contenido audiovisual, con los propios actores y con otras personas que están viendo ese mismo contenido. Por primera vez se puede establecer un diálogo con la audiencia en tiempo real” (Arrojo, 2013).

En lo que a los usuarios se refiere, el primer informe sobre redes sociales realizado por el Observatorio Nacional de las Telecomunicaciones y la Sociedad de la Información señalaba el contacto con amigos y el envío de mensajes privados como las dos actividades realizadas con más frecuencia por parte de los asiduos a este tipo de comunidades virtuales, mientras que conocer gente nueva, compartir música y compartir textos eran las prácticas menos frecuentadas (ONTSI, 2012: 13$16)$.

Junto a esto, cabe destacar que en las redes sociales conviven diferentes tipos de usuarios, entre los que se puede hablar del social controller, social media addict y youth insearch (ONTSI, 2012), mientras que otras investigaciones, como la realizada por la agencia de investigaciónForrester, habla de creadores, conversadores, críticos, carpinteros, espectadores o inactivos (Bona, 2012). Si, por otro lado, nos centramos en el perfil del multitasker-entendido aquí como el individuo que realiza otra actividad al tiempo que usa las redes sociales-, podemos hablar de cuatro usuarios diferentes: el multitasker de valor, que obtiene información al momento, expone su opinión y genera contenidos de valor; el creativo, que se sirve de la red social para desempeñar sus aficiones; el de entretenimiento, que usa este espacio para evadirse y divertirse; y el multitasker social, que se comunica con sus amigos por esta vía. De todos ellos, el perfil mayoritario es el de entretenimiento (35\%), seguido del multitasker de valor (27\%) (Havas Media Group, 2014).

Los diferentes canales de televisión, por su parte, tal y como señala Díaz-Campo tras analizar las diez cadenas de televisión con mayor número de espectadores en 2012 -RTVE, Telecinco, Antena 3, Cuatro, La Sexta, Canal Sur, TV3, Canal 9, Telemadrid y Televisión de Galicia-, tienen una presencia en las redes sociales que "puede calificarse como desigual": masiva en las plataformas más populares, "incluyendo enlaces e iconos en las portadas de sus sitios web en lugares muy visibles; pero escasa en las redes sociales de carácter más profesional y especializado, como Flickr o LinkedIn" (DíazCampo, 2014: 78). 
Entre las distintas redes sociales existentes, el V Estudio Anual de Redes Sociales señala que el promedio de canales de este tipo visitados por los internautas se situó en 3,1 en 2013, lo que supone un incremento del 17\% con respecto a 2012, siendo Facebook (94\%), Youtube (68\%) y Twitter (49\%) las más usadas y visitadas, así como aquellas a las que dedican un mayor número de horas a la semana, entre 3,32 y 4,84 (IAB, 2014).

Según el informe realizado en 2013 por la consultora Havas Media Group, más de siete millones de las personas que habitualmente realizan multitasking, lo hacen para interactuar en torno a contenidos de televisión. El mismo estudio señala la franja de 20.00 a 00.00 horas, de lunes a viernes, como el momento preferido por los internautas para ambas prácticas, siendo aquellos con edades entre 36 y 45 años quienes más intervienen en la Red de este modo (31\%), seguidos muy de cerca por el colectivo de 26 a 35 años (29\%).

Estos datos subrayan la coincidencia existente entre el prime time televisivo convencional y el que ya podemos denominar como "prime time social", siendo de 22.00 a 23.00 horas cuando la televisión registra, de lunes a viernes, sus mayores picos de audiencia, que oscilan entre el 58 y el 59,4\% (AIMC, 2014:25). Donde, sin embargo, no existe correlación es entre el perfil mayoritario de la audiencia convencional y la social. Y es que si bien son aquellos con edades comprendidas entre los 26 y los 45 años quienes más se sirven de las redes sociales para participar en programas de televisión, la audiencia tradicional mayoritaria continúa estando en aquellos de 65 y más años. En este caso, los individuos con edades entre los 35 y los 44 años constituyen el segundo grupo (19,4\%) y los de 25 a 34 años el cuarto (14,9\%) (AIMC, 2014).

\subsection{Audiencia convencional y audiencia social en las series de televisión}

Las primeras semanas de 2014 asistieron al estreno de varias series ficción españolas. El Príncipe, Galerías Velvet, Vive cantando o de Boca en Boca, entre otras, llegaron a la pequeña pantalla precedidas del éxito registrado en los audímetros por otras producciones nacionales como El tiempo entre costuras que, en su último capítulo, el 20 de enero, alcanzó un 27,8\% de cuota de pantalla. De todas ellas, El Príncipe -producida por Mediaset España en colaboración con Plano a Plano, y emitida en Telecinco-, y Galerías Velvet-creada por Bambú Producciones para Antena 3- fueron las dos series que acumularon el mayor número de espectadores, con un share entre el 21,9 y el 33,3\% en el primero de los casos, y entre el 20 y el 24\%, en el segundo (Barlovento Comunicación, 2014).

A pesar de los buenos índices de audiencia cosechados por ambas series durante su periodo de emisión, las dos compartieron liderato con otros espacios, entre los que destacaron diferentes partidos de fútbol de la Champions League emitidos por el primer canal de TVE, Telecinco y Cuatro, La voz Kids de Telecinco y la segunda edición del Telediario de la 1. 
Tabla 1. Programas de televisión con mayor cuota de pantalla entre febrero y mayo de 2014 en España

\begin{tabular}{|c|c|c|c|c|c|c|c|}
\hline \multicolumn{2}{|l|}{ Febrero } & \multicolumn{2}{|l|}{ Marzo } & \multicolumn{2}{|l|}{ Abril } & \multicolumn{2}{|l|}{ Mayo } \\
\hline Programa (share) & Canal & Programa (share) & Canal & Programa (share) & Canal & Programa (share) & Canal \\
\hline Fútbol $(37,7)$ & La 1 & Fútbol $(34,4)$ & T5 & Fútbol $(60,8)$ & La 1 & Fútbol $(61,8)$ & La 1 \\
\hline Telediario $2(31,1)$ & La 1 & La Voz Kids $(34,1)$ & T5 & Fútbol $(51,1)$ & La 1 & Fútbol $(62,1)$ & La 1 \\
\hline Fútbol (32) & La 1 & El Príncipe $(28,6)$ & T5 & Telediario2 $(45,6)$ & La 1 & Fútbol $(49,8)$ & La 1 \\
\hline La Voz Kids (30) & T5 & Fútbol $(27,5)$ & La 1 & Fútbol $(45,7)$ & La 1 & Fútbol $(39,3)$ & Cuatro \\
\hline Fútbol $(26,5)$ & A3 & El Príncipe $(27,8)$ & T5 & Fútbol $(42,2)$ & La 1 & Fútbol $(30,4)$ & Cuatro \\
\hline
\end{tabular}

Fuente: Elaboración propia a partir de datos proporcionados por Barlovento Comunicación (2014)

En lo que a audiencia social se refiere, durante los días en los que las dos series aquí analizadas estuvieron en antena, $G a-$ lerías Velvet solo lideró el ranking de audiencias sociales con sus tres primeros capítulos, mientras El Príncipe se situó entre los tres espacios con mayor número de comentarios durante todas las jornadas en antena -incluso en seis de ellas, fue el espacio más comentado, en seis el segundo y en una el tercero- (Gómez Rubio y López Vidales, 2014). No obstante, ninguna de las dos ficciones alcanza en número de comentarios a aquellos espacios con mayor actividad en las redes sociales, entre los que una vez más destacan las retransmisiones deportivas y los realities.

Tabla 2. Programas de televisión con mayor número de comentarios en las redes sociales durante los días de emisión de El Príncipe y Galerías Velvet

\begin{tabular}{|l|l|l|}
\hline Día & Programa & No de comentarios \\
\hline 29 abril & Champions: Bayern-Real Madrid (La 1) & 583.828 \\
\hline 8 abril & Champions: At. Madrid-Chelsea (La 1) & 302.467 \\
\hline 1 abril & Champions: Borussia-Real Madrid (La 1) & 297.572 \\
\hline 6 mayo & Champions: Barcelona-At. Madrid (La 1) & 288.768 \\
\hline 18 febrero & El Príncipe (Telecinco) & 225.412 \\
\hline 17 marzo & Champions: Manchester City-Barcelona (La 1) & 195.038 \\
\hline 18 marzo & Supervivientes (Telecinco) & 183.911 \\
\hline 24 marzo & Champions: Real Madrid-Schalke (La 1) & 178.793 \\
\hline 11 marzo & Supervivientes (Telecinco) & 165.288 \\
\hline
\end{tabular}




\begin{tabular}{|l|l|l|}
\hline Día & Programa & No de comentarios \\
\hline 26 mayo & Supervivientes (Telecinco) & 135.723 \\
\hline 12 mayo & Supervivientes (Telecinco) & 108.159 \\
\hline 31 marzo & Supervivientes (Telecinco) & 105.045 \\
\hline
\end{tabular}

Fuente: Elaboración propia a partir de datos de PR Noticias y Global In Media (2014)

Entre los motivos que pueden justificar el éxito social de los realities sobre otros espacios se encuentra, al margen de factores como los concursantes o el presentador, "la transmisión de estos programas en tiempo real. Para poder interactuar es preciso que la audiencia vea el espacio en directo y se genere así un discusión real e in situ" (González-Neira y QuintasFroufe, 2014: 110). Aunque esto no quiere decir que si un televidente no está viendo el programa en directo no pueda participar en las redes sociales, resulta plausible que poder intervenir en tiempo real represente un aliciente más para los habituales de esta actividad. Además, el hecho de que este tipo de espacios no requieran de una atención constante para seguir el desarrollo de su contenido supone otro incentivo que potencia el uso de las redes sociales durante la emisión del programa en directo.

\section{Metodología}

El objetivo fundamental de este trabajo es analizar la estrategia televisiva seguida en la difusión de contenidos y la actividad participativa mostrada por la audiencia que dos espacios de ficción, El Príncipe y Galerías Velvet, registraron en Facebook durante las semanas que ambas series permanecieron en antena en su primera temporada, esto es, los meses de febrero, marzo, abril y mayo de 2014. No obstante, y teniendo en cuenta que sendos espacios se unen a la red social meses antes de su estreno en la pequeña pantalla, también se estudia la actividad en Facebook de las dos ficciones españolas durante el periodo previo a su proyección en televisión, lo que nos permitirá establecer si ha habido un cambio de comportamiento en la estrategia desarrollada por sus gestores en las dos etapas, así como comprobar cuál ha sido la respuesta de los seguidores sociales de ambas series.

Para ello, el estudio se ha centrado en el análisis de la actividad experimentada en la página oficial de Facebook de las dos series desde que ambas abrieron su perfil en la red social -El Príncipe, en junio de 2013 y Galerías Velvet, en enero de 2013hasta el día siguiente de la emisión del último capítulo de la temporada: 7 y 27 de mayo de 2014, respectivamente ${ }^{2}$. La investigación se ha realizado en torno a las siguientes variables:

2 Se incluye este día en la muestra de análisis debido a que, a lo largo de toda la temporada, el horario de fin de emisión de ambas series ha tenido lugar en torno a las 00.00 horas, de modo que muchas de las reacciones de los seguidores han tenido lugar a partir de esa hora. 
(1) Número y naturaleza de los contenidos publicados en la página oficial de Facebook por parte de los canales de televisión que programan las series, tanto durante el periodo previo a su emisión en televisión, como en las semanas en las que permanecen en antena.

En este caso, el estudio comprende un análisis de los contenidos subidos a la red social por parte de los administradores de ambos sites, donde se establece la siguiente clasificación, dependiendo de los elementos textuales, visuales, audiovisuales e hipermedia empleados:

- $\quad$ Sólo texto: aquellas entradas compuestas únicamente de un enunciado escrito;

- Sólo imagen: contenidos sólo provistos de una imagen, bien sea una o varias fotografías o un vídeo cuya reproducción se realice en el propio muro de la web;

- $\quad$ Texto + imagen: donde se contabilizan aquellas entradas que contienen enunciado escrito e imagen -fotografía o vídeo-, siempre y cuando su visionado pueda realizarse en el muro de la web;

- $\quad$ Texto + enlace: se incluyen los contenidos en los que a un enunciado escrito se suma un enlace que remite a un sitio web externo, pudiendo éste pertenecer a los grupos Mediaset y Atresmedia así como a otros;

- Texto + imagen + enlace: aquellas entradas que, además de texto e imagen (fotografía o vídeo), cuentan con un enlace de una web externa, pertenezca ésta a los grupos Mediaset y Atresmedia o a otras empresas audiovisuales.

La segunda de las variables analizada es:

(2) Número y tipo de respuesta por parte de los seguidores de las series a los contenidos subidos a la red social desde que ambas producciones abren sus perfiles en Facebook hasta el día posterior a la emisión en televisión del último capítulo de la temporada.

En este punto se han contabilizado las diferentes reacciones de los internautas cada uno de los días en los que los respectivos canales han publicado algún contenido en las páginas oficiales de Facebook. Este cómputo se ha realizado teniendo en cuenta los diferentes tipos de contenidos arriba señalados, además de establecer diferencias entre los tres tipos de participación más habituales en las redes sociales:

- $\quad$ Nivel de agrado con alguna de las entradas realizadas, es decir, número de veces que han pulsado 'like’ o 'me gusta';

- $\quad$ A través de los comentarios escritos en la web;

- Mediante el número de veces que los seguidores de las series en Facebook han compartido un contenido con otros usuarios. 
Junto al objetivo principal, en las siguientes páginas también se da respuesta a:

a) cuáles fueron los contenidos que contaron con un mayor número de reacciones por parte de los usuarios de Facebook;

b) qué días subieron ambas series el mayor número de contenidos a la red social;

c) qué días acumularon una mayor respuesta por parte de los fans sociales de ambas ficciones, y de qué tipo fueron esas reacciones.

La recogida de datos se efectuó durante dos semanas completas, que fueron las posteriores a la emisión del último capítulo de cada serie: del 7 al 14 de mayo, en el caso de El Príncipe, y del 28 de mayo al 3 de junio, con Galerías Velvet. Para ello, se confeccionó una ficha en la que se contemplaba el número y naturaleza de cada uno de los contenidos subidos al muro de las páginas oficiales de Facebook de las dos producciones, el día en el que se colgaron esos contenidos y las reacciones que cada uno de ellos provocó entre los seguidores de las series en la red social.

\section{El Príncipe y Galerías Velvet en Facebook}

Las redes sociales se han convertido en canales especialmente válidos para estimular el consumo de espacios de televisión a través de la recomendación de terceros (Gallego, 2013:19), a la vez que, desde los propios programas de televisión, "cada vez más habitualmente se anima a los telespectadores a la participación en las redes sociales” (Alcolea, 2012). Las diferencias entre los responsables de la emisión de los diferentes programas de televisión que tienen reflejo en las redes sociales radica, sobre todo, en si los contenidos publicados en sus perfiles son publicados durante la jornada de su proyección en televisión, o si mantienen su actividad en la red de una forma continuada y equilibrada, por lo que a número de entradas se refiere, desde que abren sus perfiles hasta el fin de emisión televisiva de la serie.

\subsection{Un debut previo a su estreno en televisión}

En el caso de El Príncipe, aunque la serie se une a Facebook el 27 de junio de 2013 -ocho meses antes de su estreno en televisión-, no es hasta el 19 de diciembre cuando registra sus primeros contenidos. Desde ese momento y hasta la víspera del inicio de las emisiones, el 4 de febrero de 2014, la web oficial de esta red social soporta un total de 26 contenidos en 18 días. De ellos, tan sólo hay seis días en los que la página sube más de un contenido: las jornadas del 19 y 28 de diciembre y 3 de febrero, con dos entradas cada una de ellas, así como los días 20 de diciembre y 29 y 30 de enero, con tres entradas también cada uno de ellos. 
Tabla 3. Entradas registradas en la web oficial de Facebook de El Príncipe de forma previa a su emisión en televisión

\begin{tabular}{|l|l|l|l|}
\hline Año & Mes & Días con actividad en Facebook & No entradas registradas \\
\hline 2013 & Diciembre & $19,20,23,24,26,27,28$ y 31 (8 días) & 12 \\
\hline 2014 & Enero & $2,7,15,23,27,29,30$ y 31 (9 días) & 12 \\
\hline & Febrero & 3 (1 día) & 2 \\
\hline & TOTAL & 18 días & 26 entradas \\
\hline
\end{tabular}

Fuente: Elaboración propia

Todos los contenidos insertados en el muro por parte de la ficción son de promoción de la serie a través de sus personajes y también mediante un concurso en el que se regalaban 100 entradas para asistir al estreno del espacio en los cines Callao de Madrid. Para ello, los usuarios de la red únicamente tenían que indicar un adjetivo con el que calificarían a cada uno de los cuatro protagonistas de la serie ${ }^{3}$. Esta es la única vez, en este periodo previo a la emisión en televisión, que la serie organiza un concurso entre los seguidores de la serie en Facebook. La iniciativa contó con 143 respuestas, de las que 139 fueron a través de la marcación del 'me gusta', un comentario y tres seguidores que compartieron la información con otros usuarios.

En cuanto al tipo de entrada, la mayoría de las realizadas en esta primera etapa son las compuestas a base de texto e imagen -un total de 9-, junto a aquellas que, además, a estos dos elementos suman un enlace web-un total de 7-. En estos últimos casos, la mayoría de los enlaces son a otras web de Mediaset, como www.divinity.es, www.mitele.es o www.telecinco.es, así como a aquellas otras webs que hablan, por uno u otro motivo, de la serie, como www.formulatv.com o www.vertele.es. No obstante, ninguno de estos dos tipos de entradas son las que mayor aceptación cuentan entre los seguidores de la serie en Facebook, siempre teniendo en cuenta el número de comentarios, así como de veces que los usuarios han pulsado el 'like'o 'me gusta' y han compartido un contenido.

La participación en el concurso, puesta en marcha el 23 de enero de 2014, debía hacerse a través del enlace http:/ /www.telecinco.es/elprincipe/Quieres-principe-Participa-concurso-consigue_15_1736400002.html (consultado el 8 de mayo de 2014). 
Gráfico 1. Naturaleza de los comentarios colgados en la web oficial de El Príncipe antes de su estreno en televisión

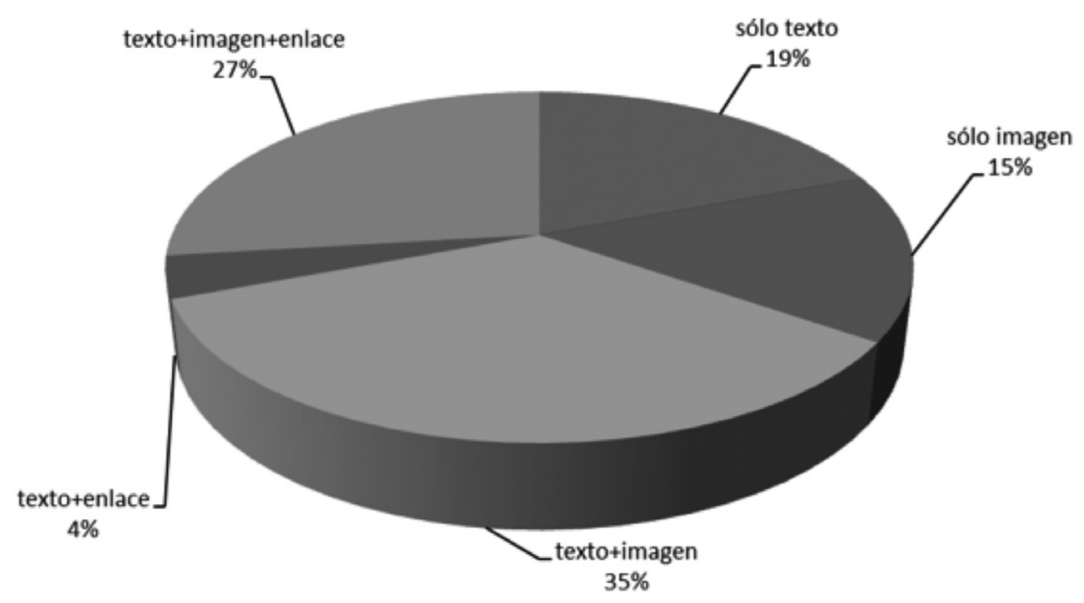

Fuente: Elaboración propia

En el caso de Galerías Velvet, la promoción de la serie a través de Facebook empieza mucho antes, incluso, que el rodaje del primer capítulo. Es en enero de 2013 cuando el espacio se une a la red social, trece meses antes de su estreno en televisión -el 16 de febrero de 2014-, y desde el primer momento, comienza a subir contenidos a la red. La primera entrada registrada en el perfil de Facebook por parte de sus gestores data del 21 de enero de 2013. Así pues, su actividad previa en Facebook difiere notablemente, al menos a nivel cuantitativo, de la experimentada por El Príncipe.

En términos generales, la promoción previa a la emisión en esta red social se desarrolla, a lo largo de esos trece meses, en un total de 92 días, que acumulan 123 entradas. Durante este tiempo, la mayoría de los días en los que la serie experimentó algún tipo de actividad en esta red social, el resultado fue de una sola entrada -un total de 67 días-, siendo 4 el número de entradas máximo registrado durante 2 jornadas. El resto de contenidos se reparten en dos y tres al día, a lo largo de 19 y 4 jornadas, respectivamente. Junto a esto, también cabe destacar que, en estos trece meses, junio de 2013 fue el que registró una mayor actividad, con 19 entradas en 10 días, mientras que enero, febrero y diciembre de 2013 fueron los menos intensos. 
Tabla 4. Entradas registradas en la web oficial de Facebook de Galerías Velvet de forma previa a su emisión en televisión

\begin{tabular}{|c|c|c|c|}
\hline Año & Mes & Días con actividad en Facebook & No entradas registradas \\
\hline \multirow{12}{*}{2013} & Enero & 21 (1 día) & 1 \\
\hline & Febrero & 24, 25 y 27 (3 días) & 5 \\
\hline & Marzo & $1,6,8,16,21,22,23,24,27$ y 31 (10 días) & 10 \\
\hline & Abril & 9, 11, 24 y 27 (4 días) & 6 \\
\hline & Mayo & $3,8,12,13,21,22,24,26,27,28$ y 29 (11 días) & 14 \\
\hline & Junio & $2,5,7,9,14,24,25,26,28$ y 29 (10 días) & 19 \\
\hline & Julio & $3,5,8,10,11,16,18$ y 23 (8 días) & 11 \\
\hline & Agosto & $6,11,12,13,21,22$ y 24 (7 días) & 10 \\
\hline & Septiembre & $8,10,11,12,13,19,21$ y 22 (8 días) & 10 \\
\hline & Octubre & $3,8,9,12,13,16,18,23,28$ y 29 (10 días) & 13 \\
\hline & Noviembre & $2,15,16,20$ y 25 (5 días) & 5 \\
\hline & Diciembre & 8 (1 días) & 1 \\
\hline \multirow{3}{*}{2014} & Enero & $2,8,14,15,23,27,28$ y 29 (8 días) & 8 \\
\hline & Febrero & $5,7,8,9,15$ y 16 (6 días) & 10 \\
\hline & TOTAL & 92 días & 123 entradas \\
\hline
\end{tabular}

Fuente: Elaboración propia

El mayor número de entradas -un total de 49- corresponde a aquellas compuestas con texto, imagen y enlace, seguidas de las provistas de texto e imagen -fotografía o vídeo-, con un total de 37. Al igual que en el caso de El Príncipe, aquellas compuestas únicamente por texto (5) y por texto más enlace (2) son las menos presentes en este periodo. Por su parte, aquellas que contienen un enlace remiten, en su mayoría, al espacio que www.mizonatv.com dedica a la ficción. 
Gráfico 2. Naturaleza de los comentarios colgados en la web oficial de Galerías Velvetantes de su estreno en televisión

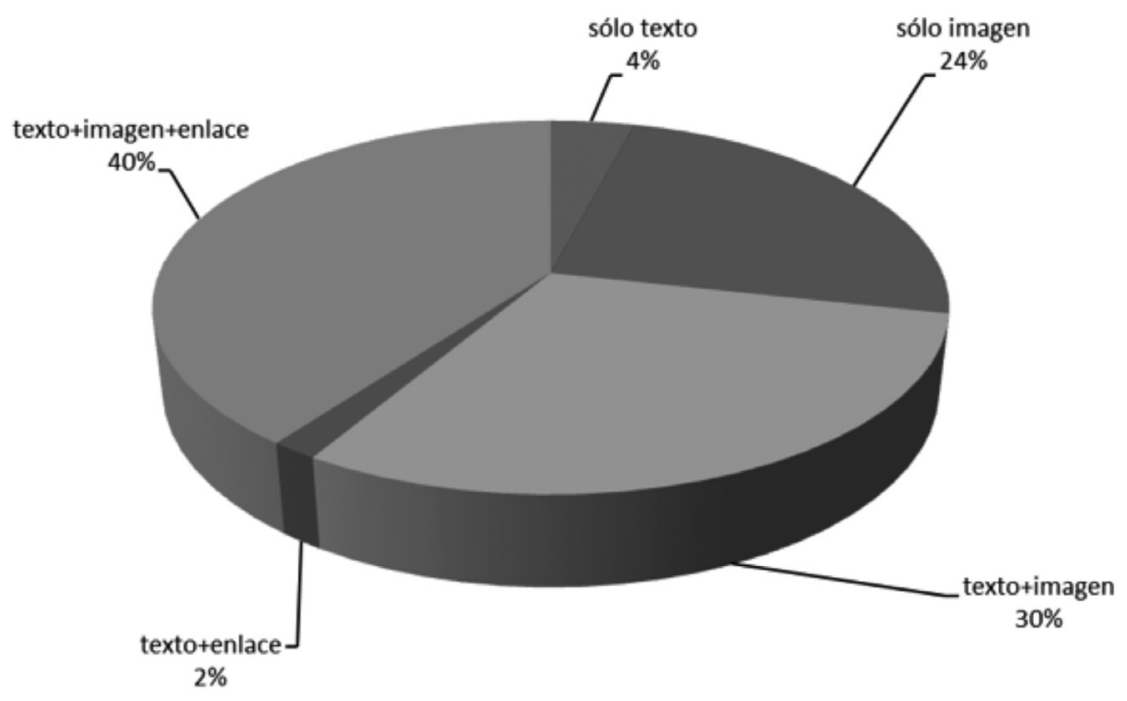

Fuente: Elaboración propia

\subsection{Combinación multipantalla y Facebook durante su emisión}

El primer capítulo de El Príncipe llegó a televisión el 4 de febrero de 2014. Desde ese día y hasta el 7 de mayo -jornada posterior a la emisión del último capítulo de la temporada- su actividad en Facebook dejó un total de 83 contenidos que fueron publicados a lo largo de 44 días: 26 durante el primer mes, febrero; 20 en marzo, otros 20 en abril y 17 en los primeros días de mayo.

Atendiendo solo a los días en los que se emitió el espacio, nos encontramos con que enero y febrero únicamente acumulan 4 contenidos publicados en cada uno de ellos, abril 9 y mayo 6. Durante estos meses, a excepción de abril, el mayor número de entradas se encuentran en la jornada siguiente a su emisión en televisión. De hecho, entre los días que alcanzan el mayor número de contenidos figuran el 5 de febrero (6) y el 7 de mayo (10), los dos correspondientes al día siguiente a la emisión del primer y último capítulo de la temporada.

Por el contrario, el día previo de su emisión en televisión se encuentra entre las jornadas que presentan el menor número de contenidos publicados. Tal y como puede verse en la tabla 5, a lo largo de los meses que la producción permaneció en antena, esos días únicamente acumularon cuatro contenidos. 
Tabla 5. Contenidos colgados en la web oficial de Facebook de El Príncipe durante los meses de su emisión en televisión

\begin{tabular}{|l|c|c|c|c|c|}
\hline Mes & $\begin{array}{c}\text { No total de conteni- } \\
\text { dos colgados }\end{array}$ & $\begin{array}{c}\text { Contenidos colga- } \\
\text { dos durante el día } \\
\text { de emisión }\end{array}$ & $\begin{array}{c}\text { Contenidos colga- } \\
\text { dos el día posterior } \\
\text { a su emisión }\end{array}$ & $\begin{array}{c}\text { Contenidos colga- } \\
\text { dos el día anterior a } \\
\text { su emisión }\end{array}$ & $\begin{array}{c}\text { Contenidos colga- } \\
\text { dos el resto de días }\end{array}$ \\
\hline Febrero & 26 & 4 & 14 & 1 & 7 \\
\hline Marzo & 20 & 4 & 7 & 1 & 8 \\
\hline Abril & 20 & 9 & 6 & 1 & 4 \\
\hline Mayo & 17 & 6 & 10 & 1 & 0 \\
\hline TOTAL & 83 & 23 & 37 & 4 & 19 \\
\hline
\end{tabular}

Fuente: Elaboración propia

En cuanto a la naturaleza de los contenidos publicados en la red, el mayor número corresponde a aquellos compuestos por texto, imagen y enlace (37), seguidos, a partes iguales, por los provistos únicamente de texto o sólo de imagen, con 18 entradas de este tipo cada una de las categorías.

Gráfico 3. Naturaleza de los comentarios publicados en la web oficial de El Príncipe durante su emisión en televisión

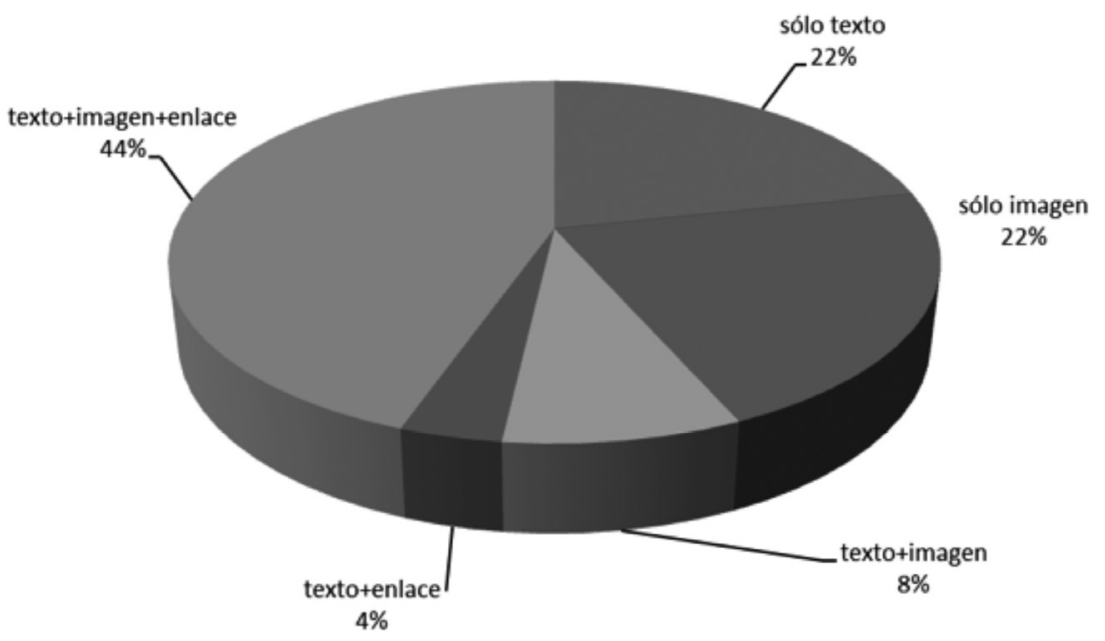

Fuente: Elaboración propia

\footnotetext{
4 La entrada registrada corresponde al 31 de marzo, día anterior a la emisión del capítulo correspondiente al 1 de abril.
} 
En el caso de Galerías Velvet, el periodo de permanencia de la serie en antena concluyó, en la red social, con un total de 85 contenidos publicados en su página oficial de Facebook en 52 jornadas. De ellos, los días en los que se subieron el mayor número de contenidos fueron los correspondientes al 26 y 27 de mayo, coincidiendo con la emisión del último capítulo de la serie, con 6 y 10 aportaciones registradas en cada una de dichas jornadas. Por el contrario, durante seis días en los que se proyectó la serie - 17 y 24 de febrero; 3, 17 y 24 de marzo; 19 de mayo-, la web no colgó ningún contenido, circunstancia que se repitió durante dos de los días posteriores a su emisión en televisión, que fueron el 18 de febrero y el 15 de abril. En lo que respecta a la víspera de su emisión en televisión, la red únicamente publicó 9 contenidos.

En general, la tónica dominante de la web es publicar uno o dos contenidos en cada una de las jornadas en las que la serie presenta alguna actividad de este tipo en Facebook. No en vano, de los 52 días en los que se realiza alguna aportación, en 13 publicaron dos contenidos, en 34 subieron un contenido y en tres días, 3.

Tabla 6. Contenidos registrados en la web oficial de Facebook de Galerías Velvetdurante su emisión en televisión (incluido 27 de mayo)

\begin{tabular}{|l|c|c|c|c|c|}
\hline Mes & $\begin{array}{c}\text { No total de conteni- } \\
\text { dos colgados }\end{array}$ & $\begin{array}{c}\text { Contenidos colga- } \\
\text { dos durante el día } \\
\text { de emisión }\end{array}$ & $\begin{array}{c}\text { Contenidos colga- } \\
\text { dos el día posterior } \\
\text { a su emisión }\end{array}$ & $\begin{array}{c}\text { Contenidos colga- } \\
\text { dos el día anterior a } \\
\text { su emisión }\end{array}$ & $\begin{array}{c}\text { Contenidos colga- } \\
\text { dos el resto de días }\end{array}$ \\
\hline Febrero & 8 & 0 & 4 & 0 & 4 \\
\hline Marzo & 21 & 3 & 4 & 3 & 11 \\
\hline Abril & 23 & 10 & 4 & 4 & 5 \\
\hline Mayo & 33 & 9 & 13 & 2 & 9 \\
\hline TOTAL & 85 & 22 & 25 & 9 & 29 \\
\hline
\end{tabular}

Fuente: Elaboración propia

Al igual que en el caso de El Príncipe, la serie de Antena 3 también optó de forma mayoritaria por las aportaciones a base de texto, imagen y enlace: un total de 37 de los contenidos publicados en Facebook fueron de este tipo, seguidos de aquellos compuestos solo de imagen (25) y de texto e imagen (21). Estas tres modalidades monopolizan los contenidos publicados durante el periodo de emisión de la ficción, puesto que únicamente hubo dos entradas de solo texto y ninguna que contuviera texto y enlace. 
Gráfico 4. Naturaleza de los comentarios colgados en la web oficial de Galerías Velvet durante su emisión en televisión

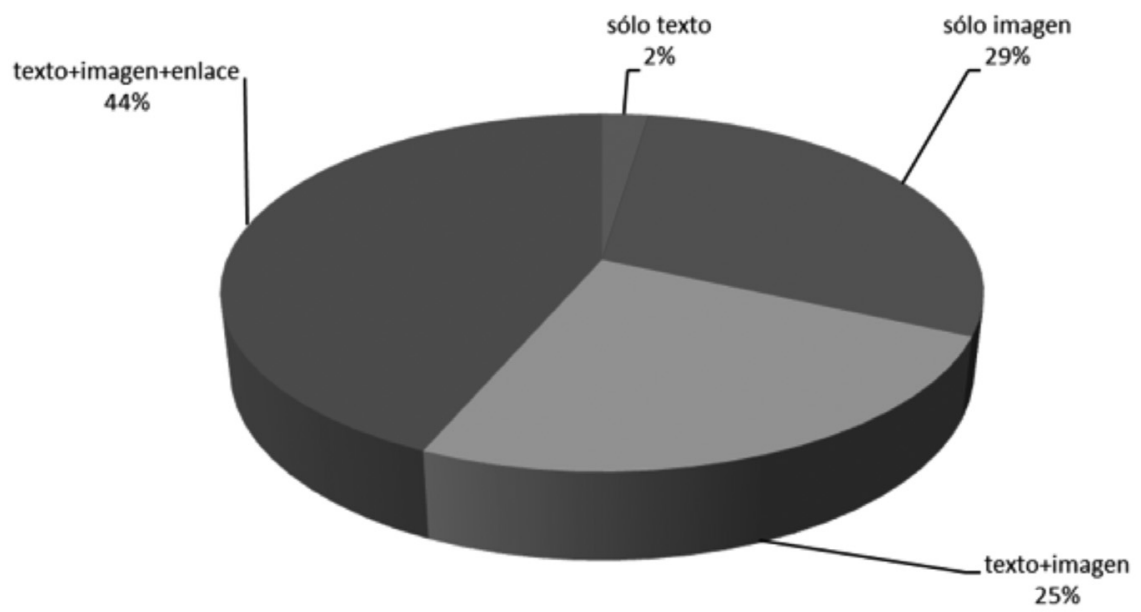

Fuente: Elaboración propia

\section{La respuesta de la audiencia social}

A través de Facebook, los seguidores sociales de un programa de televisión pueden opinar, intercambiar y compartir aquellos contenidos que más les gustan y/o interesan. Sin embargo, ¿qué tipo de contenidos son los que provocan una mayor respuesta y participación entre ellos? La información obtenida a través de esta pregunta es primordial para comprobar si, en ambas ficciones, los contenidos publicados por los gestores de sus perfiles en la red son los deseados por sus "fans" sociales (García Avilés, 2012:437). Otra de las cuestiones fundamentales para determinar la efectividad de la estrategia desarrollada por los canales de televisión en Facebook está en conocer si la participación de los usuarios en esta comunidad virtual se concentra especialmente en aquellos días que coinciden con la emisión de las series en televisión o si, por el contrario, la actividad de los seguidores viene determinada en función de la naturaleza de los contenidos y no tanto del día en que aparecen en el muro virtual de las ficciones.

\subsection{Participación antes de su emisión en televisión}

En la página oficial de Facebook de El Príncipe, cabe destacar que aquellas entradas compuestas únicamente por una imagen son las que sumaron el mayor número de comentarios, de 'like' y los más compartidos: solo 4 entradas de este tipo superaron los 3.000 ' like', los 170 comentarios y los 360 'shared'. Por el contrario, las inserciones a cargo de texto y enlace y aquellas otras portadoras únicamente de texto son las menos visitadas, comentadas y recomendadas por los usuarios. Y 
ello a pesar de que la presencia de enlaces en los contenidos publicados "favorece una estrategia transmedia al contar con otro soporte, la web, que recoge información extra sobre el programa, incluso el seguimiento de la audiencia social" (Quintas-Froufe y González-Neira, 2014).

Tabla 7. Naturaleza de las entradas en la web oficial de Facebook de El Príncipe durante el periodo previo a su emisión en televisión

\begin{tabular}{|l|c|c|c|c|}
\hline & Entradas & Like & Comentarios & Shared \\
\hline Sólo texto & 5 & 711 & 40 & 24 \\
\hline Solo imagen & 4 & 3.007 & 176 & 368 \\
\hline Texto + imagen & 9 & 1.157 & 69 & 201 \\
\hline Texto + enlace & 1 & 32 & 5 & ---- \\
\hline Texto + imagen + enlace & 7 & 1.296 & 38 & 141 \\
\hline TOTAL & 26 & 6.203 & 328 & 734 \\
\hline
\end{tabular}

Fuente: Elaboración propia

De todos los contenidos comprendidos en este periodo, el que más reacciones consiguió por parte de los internautas fue el que presentó fotos de los personajes correspondientes a Mati, Raquel y Khaled, del 3 de febrero de 2014 -víspera de la emisión del primer capítulo de la temporada-, con 1.912 personas que se sumaron al 'me gusta'. Se trata, además, de una entrada que solo comprende imagen (fotos de esos personajes cogidas del "álbum de la serie"), y en los que, si quieres saber más de esos personajes, tienes que pinchar sobre la foto. Si nos centramos en el que más comentarios acumuló en esta primera etapa de análisis - un total de 94-, en este caso es una foto de cubierta, también solo imagen, puesta el 2 de enero, siendo esta misma foto, precisamente, la que más se compartió, un total de 164 veces.

En el caso de Galerías Velvet, los contenidos a base de texto e imagen son los que han contado con una mayor respuesta por parte de los internautas, tanto a la hora de mostrar su agrado con el contenido publicado, con más de 5.000 personas que dieron al 'me gusta' en alguna entrada de este tipo, como a la hora de realizar algún comentario y de compartirlo. De hecho, cuatro entradas de este tipo -realizadas los días 10 y 16 de julio, 6 de agosto y 8 de octubre de 2013-, y que ilustran diferentes momentos del rodaje de la serie- son las que acumularon el mayor número de 'me gusta' y de comentarios, mientras que dentro de los contenidos más compartidos se encuentra uno registrado el 16 de noviembre y que muestra un tráiler de la serie, compartido un total de 55 veces, así como otro del 8 de febrero de 2014, también de un vídeo promocional, compartidos por 51 personas. 
Tabla 8. Naturaleza de las entradas en la web oficial de Facebook de Galerías Velvet durante el periodo previo a su emisión en televisión

\begin{tabular}{|l|c|c|c|c|}
\hline & Entradas & Like & Comentarios & Shared \\
\hline Sólo texto & 5 & 132 & 5 & 18 \\
\hline Solo imagen & 30 & 1.127 & 44 & 57 \\
\hline Texto + imagen (foto o vídeo) & $21+16$ & $4.248+723$ & $122+51$ & $114+180$ \\
\hline Texto + enlace & 2 & 56 & 3 & 3 \\
\hline Texto + imagen + enlace & 49 & 1.626 & 44 & 412 \\
\hline TOTAL & 123 & 7.912 & 269 & 484 \\
\hline
\end{tabular}

Fuente: Elaboración propia

\subsection{Participación durante su emisión en televisión}

La superioridad numérica de El Príncipe en los contenidos a base de texto, imagen y enlace, se traduce también en el mayor número de reacciones provocadas en la audiencia: esta modalidad de contenido acapara el mayor número de veces que los seguidores han pulsado 'me gusta', así como de comentarios escritos. Sin embargo, no son los contenidos más compartidos entre los usuarios, lugar que quedó reservado para aquellas entradas con texto e imagen: las siete entradas de este tipo fueron compartidas 3.700 veces, entre los que destaca uno de los publicados el 8 de abril -jornada que coincide con la emisión del espacio en televisión-, en los que se cuelgan cuatro fotos de diferentes momentos de la serie.

Llama también la atención que los siete contenidos de texto e imagen hicieran que los seguidores dieran más de 32.800 veces al 'me gusta'. En este punto, nuevamente nos encontramos con el anterior contenido mencionado, uno de los subidos a Facebook el 8 de abril, que acumuló más de 6.400 'like’. En esta misma línea, cabe subrayar que, a pesar de que el mayor número de comentarios fueron registrados en las entradas de texto, imagen y enlace, el mayor número de comentarios fueron realizados el 6 de mayo -coincidiendo con la emisión del último capítulo-, de la mano de un contenido a cargo de texto e imagen, que sumó más de 1.500 comentarios. A continuación, se sitúa otro de solo texto, subido el 5 de febrero, es decir, al día siguiente del estreno de la serie en televisión, con 761 comentarios. Se trata además, de un contenido en el que, precisamente, se incita a los seguidores del espacio a su participación en la red a través de esta modalidad 5 .

5 El contenido recoge textualmente “Bueno bueno ¡queremos comentarios!”. 
Tabla 9. Naturaleza de contenidos en la web oficial de Facebook de El Príncipe durante su emisión en televisión (incluido 7 de mayo)

\begin{tabular}{|l|c|c|c|c|}
\hline & Entradas & Like & Comentarios & Shared \\
\hline Sólo texto & 18 & 42.042 & 2.625 & 2.509 \\
\hline Solo imagen & 18 & 56.786 & 1.374 & 2.490 \\
\hline Texto + imagen & 7 & 32.872 & 2.703 & 3.700 \\
\hline Texto + enlace & 3 & 2.427 & 77 & 353 \\
\hline Texto + imagen + enlace & 37 & 74.666 & 3.028 & 1.647 \\
\hline TOTAL & 83 & 208.793 & 9.807 & 10.699 \\
\hline
\end{tabular}

Fuente: Elaboración propia

Respecto a los días en los que los seguidores participan más activamente en la red social se encuentran aquellos que coinciden con el día después de la emisión en televisión de la serie, algo debido, probablemente, a que la serie finaliza en torno a las 00.00 horas, de modo que muchos de los espectadores esperan a que el espacio concluya para participar, de una u otra forma, en la red social. De todas las respuestas acumuladas durante los meses que el espacio permanece en antena, la actividad de los fans sociales a los contenidos publicados al día siguiente de su emisión concentran el 43,7\% del total de las reacciones registradas, seguidas de aquellas realizadas durante el día de la emisión en televisión (40,4\%). Por el contrario, las que tienen lugar el día anterior a la emisión tan solo acumulan el 4,7\%, mientras que las realizadas el resto de las jornadas representan el $11,2 \%$.

Tabla 10. Respuestas de los seguidores web oficial de Facebook de El Príncipe durante su emisión en televisión

\begin{tabular}{|c|c|c|c|c|c|c|c|c|}
\hline \multicolumn{3}{|c|}{ Día de emisión } & \multicolumn{3}{c|}{ Día posterior } & \multicolumn{3}{c|}{ Día anterior } \\
\hline Like & Com & Shared & Like & Com & Shared & Like & Com & Shared \\
\hline 81.942 & 4.012 & 6.731 & 93.965 & 4.042 & 2.387 & 10.148 & 233 & 413 \\
\hline \multicolumn{3}{|c|}{ TOTAL 92.685 } & \multicolumn{3}{c|}{ TOTAL 100.394 } & \multicolumn{3}{c|}{ TOTAL 10.794 } \\
\hline
\end{tabular}

Fuente: Elaboración propia

En Galerías Velvet, los contenidos que registraron mayor actividad fueron aquellos con texto e imagen: los 21 contenidos de este tipo que publicó la producción acumularon el mayor número de 'me gusta' -alrededor de 14.400-, así como el 
mayor número de comentarios -más de 1.600-, además de ser los contenidos más compartidos. Entre ellos, se encuentran cuatro de los publicados el 25 de febrero, 25 de marzo, 26 de mayo y 27 de mayo, que superan los 1.300 'like' cada uno de ellos. Se trata, en todos los casos, de contenidos compuestos por texto e imagen subidos a la red social el día siguiente a la emisión de la serie en televisión, a excepción de la entrada correspondiente al 26 de mayo, que tiene lugar durante la jornada de emisión del último capítulo de la temporada. Esos contenidos son también los que suscitaron un mayor número de comentarios y algunos de los más compartidos.

Tabla 11. Naturaleza de las entradas en la web oficial de Facebook de Galerías Velvet durante su emisión en televisión (incluido 27 mayo)

\begin{tabular}{|l|c|c|c|c|}
\hline & Entradas & Like & Comentarios & Shared \\
\hline Sólo texto & 2 & 1.168 & 14 & 96 \\
\hline Solo imagen (foto o vídeo) & 25 & 8.698 & 341 & 619 \\
\hline Texto + imagen (foto o vídeo) & 21 & 14.379 & 1.679 & 631 \\
\hline Texto + enlace & 0 & 0 & 0 & 0 \\
\hline Texto + imagen + enlace & 37 & 11.917 & 623 & 310 \\
\hline TOTAL & 85 & 36.162 & 2.657 & 1.656 \\
\hline
\end{tabular}

Fuente: Elaboración propia

Aquí, también el mayor número de respuestas por parte de la audiencia se encuentra al día siguiente de la proyección de la serie en la pequeña pantalla. De hecho, estos días acumulan el 40,6\% del total de la actividad registrada por la serie durante su permanencia en Antena 3, seguido de aquellos que coinciden con su emisión (30,9\%), mientras que las respuestas efectuadas en las jornadas anteriores a su programación representan el 6,3\% de la actividad. El resto de días de la temporada suman el 22,2\% de las reacciones de los fans sociales.

Tabla 12. Respuestas de los seguidores web oficial de Facebook de Galerías Velvetdurante su emisión en televisión (incluido el 27 de mayo)

\begin{tabular}{|c|c|c|c|c|c|c|c|c|}
\hline \multicolumn{3}{|c|}{ Día de emisión } & \multicolumn{3}{c|}{ Día posterior } & \multicolumn{3}{c|}{ Día anterior } \\
\hline Like & Com & Shared & Like & Com & Shared & Like & Com & Shared \\
\hline 11.625 & 559 & 354 & 14.296 & 1.630 & 511 & 2.283 & 95 & 191 \\
\hline \multicolumn{3}{|c|}{ TOTAL 12.538 } & \multicolumn{3}{c|}{ TOTAL 16.437 } & \multicolumn{3}{c|}{ TOTAL 2.569 } \\
\hline
\end{tabular}

Fuente: Elaboración propia 


\section{Discusión de los resultados y conclusiones}

La estrategia que siguen las cadenas de televisión en las distintas redes sociales fomenta la participación del público en las series de ficción con el objetivo de aumentar la audiencia total del producto. En consecuencia, las empresas audiovisuales comienzan a publicitar y promocionar las series, especialmente las de producción propia, meses antes de su emisión en la pequeña pantalla, mediante la apertura de perfiles propios de cada espacio en las redes sociales. Con ello crean expectación y motivan al público para visionar posteriormente la serie concreta. Se trata de una estrategia muy extendida en los últimos años en el lanzamiento previo de otros contenidos audiovisuales y mediáticos relacionados con el entretenimiento, como es el caso de los videojuegos.

Esta estrategia contempla el empleo de características "crossmedia” y "transmedia" en la construcción de los formatos, lo que favorece las posibilidades de compartir los contenidos de estos espacios de ficción a través de múltiples plataformas mediáticas y partiendo de historias o piezas diferenciadas, ligadas entre sí, en cuyo desarrollo y difusión participa el usuario-espectador de forma activa.

Este ha sido el modus operandi de las series aquí analizadas. Sin embargo, aunque ambas se unen a Facebook meses antes de su estreno en la pequeña pantalla, la estrategia desarrollada durante los primeros meses por cada de ellas es diferente: mientras El Príncipe abre su perfil en la red social en junio de 2013 -ocho meses antes de su estreno en televisión-, el primer contenido que publica la página se registra en diciembre de 2013, mes y medio antes de la emisión del primer capítulo; Galerías Velvet, por su parte, se une a Facebook trece meses antes de su emisión en televisión y, desde ese momento, comienza a publicar contenidos en el muro de la red.

Esto contribuye a que, durante el periodo previo a la emisión de ambas ficciones en televisión, Galerías Velvet cuente con una mayor actividad en lo que se refiere al número de días y de contenidos que los administradores del site subieron a la red social. Durante ese periodo previo a la proyección de ambos espacios en televisión, ambas series se decantan de forma mayoritaria por aquellas entradas compuestas por texto e imagen y las que contienen texto, imagen y un enlace web. La primera de las tipologías es la más presente en El Príncipe-un 35\% de los contenidos colgados son de este tipo-, mientras que la segunda es la más habitual en Galerías Velvet (40\%).

Sin embargo, en esos meses, este tipo de contenidos no son los que provocan el mayor número de reacciones entre los seguidores de los dos espacios en Facebook: los fans sociales de la serie de Telecinco muestran una mayor respuesta ante aquellos contenidos compuestos solo por una imagen, que suponen el 15\% de las entradas registradas en ese periodo, mientras que los seguidores de la producción de Antena 3 prefieren aquellos compuestos por texto e imagen, que es el segundo tipo de contenidos más publicados, representando al $30 \%$ de total de entradas. 
Tras el estreno en televisión de las dos series, la estrategia desarrollada por los canales responsables de su emisión presenta pocas diferencias. Ambas continúan decantándose, para su publicación en Facebook, por contenidos, sobre todo, visuales y audiovisuales, concentrando, en ambas series, más de la mitad de los contenidos publicados durante el periodo de emisión en televisión.

Asimismo, son este tipo de entradas las que cuentan con una mayor respuesta en la red social por parte de sus seguidores que, sobre todo, se traduce de forma mayoritaria en la marcación del 'me gusta', frente a dejar un comentario o compartir un contenido. Se trata, además, de una participación especialmente concentrada en las jornadas de emisión en televisión y día siguiente, con porcentajes que superan el $80 \%$ en El Príncipe y el $70 \%$ en Galerías Velvet. Esto pone de manifiesto que los seguidores sociales de estas ficciones prefieren participar en la red durante el espacio de tiempo más próximo a su proyección en televisión.

Según los resultados obtenidos, puede afirmarse que la participación de la audiencia en las redes sociales se acrecienta cuando los contenidos que figuran en las webs son de carácter audiovisual, especialmente imágenes y vídeos, máxime cuando presentan a los protagonistas de las series, están relacionados con el perfil de los personajes que intervienen, narran anécdotas del rodaje o proporcionan algún tipo de información extra. Estas piezas crean "historias" relacionadas con el contenido de cada capítulo de la serie, pero al tiempo tienen un desarrollo propio gracias a la intervención del usuario, lo que genera la percepción por parte de éste de un mayor conocimiento de los detalles de la producción y la trama general. La sensación de "ventaja" sobre los espectadores televisivos también supone un aliciente del individuo para su participación efectiva en las redes sociales.

La construcción de la audiencia social, por tanto, representa aquí el primer paso para asegurarse el éxito de la serie en su emisión televisiva y a través de otros dispositivos. Representa, de un lado, un punto importante en la consecución del engagement o enganche con el participante, y por otro, procura asegurar el "longtail" de las nuevas producciones de formatos televisivos.

\section{Referencias bibliográficas}

AIMC (2014): Resumen General del EGM. Octubre de 2013 - Mayo de 2014.

AIMC (2014): Marco General de los Medios en España.

Alcolea Díaz, G. (2012): "Estrategias televisivas en redes sociales: los twittersodios, un estudio de caso sobre consumo juvenil”. Actas del IV Congreso Internacional Latina de Comunicación Social. Universidad de La Laguna. Disponible en: http://www.ull.es/publicaciones/latina/12SLCS/2012_actas/145_Alcolea.pdf [Consultado el 20/X/14]. 
Alonso González, M. (2014): “Audiencia Social: el telespectador comienza a participar en los contenidos televisivos”, Ámbitos, n. 25. Disponible en: http://ambitoscomunicacion.com/2014/audiencia-social-el-telespectador-comienza-aparticipar-en-los-contenidos-televisivos/ [Consultado el 1/XI/14].

Anderson, C. (2004): “The long tail”, Wired Magazine. Disponible en: http://archive.wired.com/wired/archive/12.10/ tail.html [Consultado el 27/11/14].

Arrojo, M. J. (2013): “La creatividad en el diseño de nuevos procesos de comunicación. La Televisión Social como ejemplo del cambio de modelo". Actas del V Congreso Internacional Latina de Comunicación Social. Universidad de La Laguna. Disponible en: http://www.ull.es/publicaciones/latina/13SLCS/2013_actas/063_Arrojo.pdf [Consultado el 15/X/14].

Barlovento Comunicación (2014): El comportamiento de la audiencia televisiva. Informe mensual (febrero, marzo, abril y mayo).

Barrientos Bueno, M. (2013): “La convergencia y la segunda pantalla televisivas: el caso de Isabel (TVE)”. I Congreso Internacional de Comunicación y Sociedad Digital. Disponible en: http://reunir.unir.net/handle/123456789/1731 [Consultado el 20/X/14].

Bona, Y. (2012): "Perfiles de usuarios en las redes sociales: del inactivo al súper usuario", UOC, Elperiodico.com. Disponible en: http://uoc2012.elperiodico.com/2012/06/27/perfiles-de-usuarios-en-las-redes-sociales-del-inactivo-al-\%E2\% 80\%98 super-usuario\%E2\%80\%99/ [Consultado el 25/XI/14].

Díaz-Campos, J. (2014): “Las cadenas de televisión españolas en Internet: un estudio sobre la calidad de sus sitios web”, Estudios sobre el Mensaje Periodístico, vol. 20, n. 1, p. 6783. Disponible en: http://revistas.ucm.es/index.php/ ESMP/article/viewFile/45219/42559 [Consultado el 20/X/14].

Ferreras Domínguez, E. M. (2011): "La estrategia de la corporación EITB (Euskal Irratia Telebista) en Facebook y Twitter", Razón y Palabra, n. 76. Disponible en: http://www.redalyc.org/pdf/1995/199519981018.pdf [Consultado el 22/X/14].

Gallego, F. (2013): “Social TV analytics: nuevas métricas para una nueva forma de ver televisión”, INDEX Comunicación, n. 3, pp. 13-39.

García Avilés, J. A. (2012): "Roles of audience participation in multiplatform television: From fans and consumers, to collaborators and activists", Journal of Audiencie \& Reception Studies, vol. 9, pp. 429-447. Disponible en: http://www.participations.org/Volume\%209/Issue\%202/24\%20Garcia-Aviles.pdf [Consultado el 2/XI/14].

García Mirón, S. (2011): “Televisión y redes sociales: presencia de la estrategia 3.0 de Antena 3 en Facebook”, en Ortega, F. y Cardeñosa, L. (eds.): Nuevos medios, nueva comunicación. Actas del II Congreso Internacional Comunicación 3.0. Salamanca: USAL, pp. 85-103. Disponible en: http://comunicacion3punto0.files.wordpress.com/2011/05/ comunicacion3punto0libroactas2010.pdf [Consultado el 2/IX/14]. 
Gómez Rubio, L. y López Vindales, N. (2014): "Participación de la audiencia a través de las redes sociales en las series de televisión: análisis de los casos de El Príncipe y Galerías Velvet en Facebook”, en González Vallés, J. E, y Valderrama Santomé, M. (coords.): Comunicación actual: Redes sociales y lo 2.0 y 3.0. Madrid: McGraw Hill (en edición).

González Neira, A. y Quintas-Froufe, N. (2014): "Audiencia tradicional frente a audiencia social: un análisis comparativo en el prime-time televisivo". Revista Mediterránea de Comunicación, vol. 5 (1), pp. 105-121. Disponible en: http://mediterranea-comunicacion.org/ [Consultado el 20/X/14].

Havas Media Group (2014): TV Social; tú emite, que yo comento. Disponible en: http://www.lacomunidadpublicitaria.com/ files/field/file/hmg_social_tv_aedemo_tv_2013.pdf [Consultado el 15/X/14].

IAB (2014): V Estudio anual de redes sociales. Disponible en: http://www.iabspain.net/wp-content/uploads/downloads/2014/04/V-Estudio-Anual-de-Redes-Sociales-versi\%C3\%B3n-reducida.pdf [Consultado el 14/VI/14].

ONTSI (2014): La sociedad en Red. Informe anual 2013. Madrid: Ministerio de Industria, Energía y Turismo.

Parra, P., Gordo, A. J. y D’Antonio, S. A. (2014): “La investigación social aplicada en redes sociales. Una innovación metodológica para el análisis de los «Me gusta» en Facebook", Revista Latina de Comunicación Social, n. 69, pp. 195-212. Disponible en: http://www.revistalatinacs.org/069/paper/1008_UCM3/11p.html [Consultado el 25/XI/14].

Portaltic (2014): “Infografía de usuarios de redes sociales a junio de 2014". Disponible en: http://www.europapress.es/portaltic/socialmedia/noticia-infografia-usuarios-redes-sociales-junio-2014-20140618142429.html [Consultado el 20/X/14].

Quintas-Froufe, N. y González Neira, A. (2014): “Audiencias activas: participación de la audiencia social en la televisión”, Comunicar, n. 43. Disponible en: http://www.revistacomunicar.com/index.php?contenido=detalles\&numero=43\&articulo=43-2014-08 [Consultado el 1/IX/14]. 